\title{
Magnetic-field-dependent quasiparticle dynamics of nanowire single-Cooper-pair transistors
}

\author{
Jasper van Veen, ${ }^{1,2}$ Alex Proutski, ${ }^{1,2}$ Torsten Karzig, ${ }^{3}$ Dmitry I. Pikulin, ${ }^{3}$ Roman M. Lutchyn, ${ }^{3}$ Jesper Nygård, ${ }^{4}$ \\ Peter Krogstrup, ${ }^{5}$ Attila Geresdi, ${ }^{1,2}$ Leo P. Kouwenhoven, ${ }^{1,2,6}$ and John D. Watson ${ }^{6, *}$ \\ ${ }^{1}$ QuTech, Delft University of Technology, 2600 GA Delft, The Netherlands \\ ${ }^{2}$ Kavli Institute of Nanoscience, Delft University of Technology, 2600 GA Delft, The Netherlands \\ ${ }^{3}$ Station Q, Microsoft Corporation, Santa Barbara, California 93106-6105, USA \\ ${ }^{4}$ Center for Quantum Devices, Niels Bohr Institute, University of Copenhagen, \\ 2100 Copenhagen, Denmark \\ ${ }^{5}$ Center for Quantum Devices and Microsoft Quantum Lab Copenhagen, Niels Bohr Institute, \\ University of Copenhagen, 2100 Copenhagen, Denmark \\ ${ }^{6}$ Microsoft Station Q Delft, Delft University of Technology, 2600 GA Delft, The Netherlands
}

(Received 3 July 2018; published 9 November 2018)

\begin{abstract}
Parity control of superconducting islands hosting Majorana zero modes (MZMs) is required to operate topological qubits made from proximitized semiconductor nanowires. We test this control by studying parity effects in hybrid InAs-Al single-Cooper-pair transistors (SCPTs) to evaluate the feasibility of this material system. In particular, we investigate the gate-charge modulation of the supercurrent and observe a consistent $2 e$-periodic pattern indicating a general lack of low-energy subgap states in these nanowires at zero magnetic field. In a parallel magnetic field, an even-odd pattern develops with a gate-charge spacing that oscillates as a function of field demonstrating that the modulation pattern is sensitive to the presence of a single bound state. In addition, we find that the parity lifetime of the SCPT decreases exponentially with magnetic field as the bound state approaches zero energy. Our work shows that aluminum is the preferred superconductor for future topological qubit experiments and highlights the important role that quasiparticle traps and superconducting gap engineering would play in these qubits. Moreover, we demonstrate a means by which bound states can be detected in devices with superconducting leads.
\end{abstract}

DOI: 10.1103/PhysRevB.98.174502

\section{INTRODUCTION}

The interplay of charging energy $E_{C}$ and the superconducting gap $\Delta$ leads to the surprising result that the electrical transport in a mesoscopic superconducting island containing a macroscopic number of electrons is sensitive to the addition or removal of a single electron [1-4]. This parity effect has been extensively studied in $\mathrm{Al}-\mathrm{AlO}_{x}$ SCPTs by measurements of the $2 e$-periodic gate-charge modulation of the Coulomb peak spacing, the ground state charge, and the switching current [5-13]. In recent experiments, the presence of MZMs in hybrid semiconductor-superconductor nanowires was inferred from the field-induced $1 e$ Coulomb blockade periodicity, illustrating the utility of this periodicity in understanding the low-energy spectrum of mesoscopic superconducting islands [14-21]. In contrast with these previous studies which utilized devices with normal metal leads, we investigate parity effects in gate-tuneable nanowire SCPTs

\footnotetext{
*john.watson@microsoft.com
}

Published by the American Physical Society under the terms of the Creative Commons Attribution 4.0 International license. Further distribution of this work must maintain attribution to the author(s) and the published article's title, journal citation, and DOI. which have superconducting leads by studying the junction gate, temperature, and parallel magnetic field dependence of the switching current modulation. These experiments not only give insights into quasiparticle dynamics but also represent a step towards implementing recent Majorana-based qubit proposals which require Josephson coupling to the leads to enable parity-to-charge conversion for MZM manipulation and readout [22-26].

The Hamiltonian of a SCPT consists of three terms: $H=$ $H_{C}+H_{J}+H_{\mathrm{BCS}}$. The Coulomb term, $H_{C}=E_{C}\left(n-n_{g}\right)^{2}$, stabilizes the excess charge $n$ on the island which can be changed by varying the gate charge $n_{g}$. The effective charging energy $E_{C}=e^{2} / 2 C$ is given in terms of the electron charge $e$ and a generalized capacitance $C$ that takes into account the geometric capacitance and possible renormalization effects due to tunneling of quasiparticles [27-31]. The Josephson term for symmetric junctions $H_{J}=-E_{J} \cos (\phi / 2) \sum_{n}|n\rangle\langle n+2|+$ H.c., with $E_{J}$ the Josephson energy and $\phi$ the superconducting phase difference across the island, couples adjacent, equalparity states and results in energy level anticrossings when states with the same parity are degenerate. The third term describes the spectrum of the gapped BCS quasiparticles resulting in an energy offset $\Delta$ for the odd ground state due to an unpaired electron in the superconductor. Figure 1(a) shows the resulting band structure of a SCPT. The corresponding gate-charge modulation of the critical current is shown in 


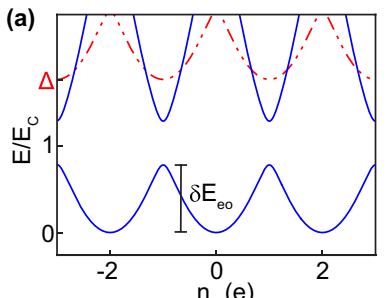

(b)

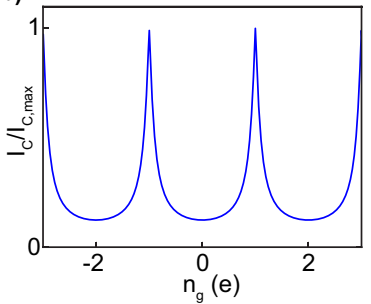

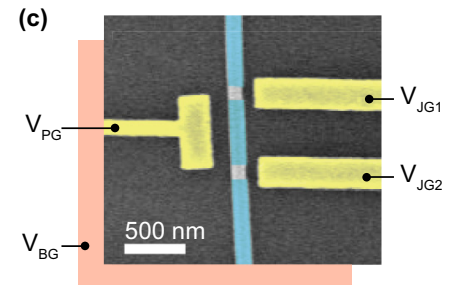

(d)

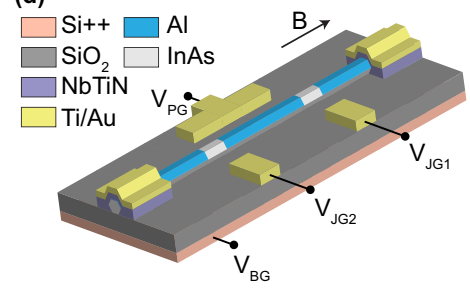

FIG. 1. Theoretical background and device layout. (a) The band structure of a SCPT as a function of gate charge $n_{g}$ for $\phi=0$, $\Delta / E_{C}=1.5$, and $E_{J} / E_{C}=0.25$. The charge dispersion of the odd parity branch (in red) is displaced from the even parity branch (in blue) by the superconducting gap $\Delta$ and $\delta n_{g}=1$. The amplitude of the ground state charge dispersion is denoted by $\delta E_{e o}(\mathrm{~b})$. The corresponding critical current modulation as a function of $n_{g}$. (c) A false-colored scanning electron micrograph of a nanowire SCPT. The etched regions in the $\mathrm{Al}$ shell define the junctions and the island. By applying voltages to the electrostatic gates, we can tune the chemical potential (with $V_{B G}$ ), the junction transparency (with $V_{J G 1,2}$ ), and the charge occupation of the island (with $V_{P G}$ ). (d) Three-dimensional device schematic. The nanowire is deterministically placed on top of $\mathrm{a} \mathrm{SiO}_{2} / \mathrm{Si}++$ substrate. It is then contacted by a stack of NbTiN and $\mathrm{Ti} / \mathrm{Au} 1 \mu \mathrm{m}$ away from the etched regions. Finally, the local gates are deposited. The arrow indicates the direction of the magnetic field for the data presented in Fig. 4.

Fig. 1(b). We denote the amplitude of the (even) ground state charge dispersion $E_{\mathrm{gs}}\left(n_{g}\right)$ with $\delta E_{\mathrm{eo}}=E_{\mathrm{gs}}\left(n_{g}=1\right)-$ $E_{\mathrm{gs}}\left(n_{g}=0\right)$. When $\Delta>\delta E_{\mathrm{eo}}$ the ground state is always even. Consequently, the switching current modulation will be $2 e$ periodic at $T=0$ in this simple model.

Quasiparticle poisoning, however, affects this $2 e$-periodic modulation. Previous studies have illustrated three important timescales, namely the poisoning rate $\Gamma_{\text {in }}$ at which quasiparticles in the lead tunnel to the island, the nonequilibrium unpoisoning rate $\Gamma_{\text {out }}^{\text {neq }}$ at which nonequilibrium quasiparticles on the island tunnel out to the leads, and the relaxation rate $1 / \tau$ at which nonequilibrium quasiparticles on the island relax to the gap edge or subgap states [32-35]. While the relaxation is important for the quasiparticle dynamics, the thermodynamics of the system can be described by equilibrium poisoning and unpoisoning rates $\Gamma_{\text {in }}$ and $\Gamma_{\text {out }}$ alone; therefore, we leave the implications of relaxation in our devices to the discussion section below. The ratio $\Gamma_{\text {in }} / \Gamma_{\text {out }}$ gives the relative occupation between the even and odd parity states in equilibrium $p_{\text {odd }} / p_{\text {even. }}$. If $\Gamma_{\text {in }} / \Gamma_{\text {out }} \approx 1$ as is expected to occur at high temperature, the switching current modulation deviates from $2 e$ periodicity and exhibits a $1 e$ periodicity instead.

Figure 1(c) presents a scanning electron micrograph (SEM) of one of our SCPTs, and a three-dimensional schematic of the

device is shown in Fig. 1(d). The SCPTs are fabricated from InAs nanowires covered with a thin aluminium shell on two of their facets. It has been shown that this material combination results in a hard, induced superconducting gap in the nanowire $[36,37]$. The aluminium shell is etched in two regions along the nanowire in order to define the island together with the two Al-InAs-Al Josephson junctions. The wire is contacted $1 \mu \mathrm{m}$ away from each junction by $\mathrm{NbTiN} / \mathrm{Ti} / \mathrm{Au}$ contacts which are expected to act as quasiparticle traps due to the presence of normal metal and the large subgap density of states in NbTiN [38]. Previous studies have shown the effectiveness of such traps to reduce the quasiparticle density $[7,35,39]$. Voltages $V_{J G 1}$ and $V_{J G 2}$ applied to the side gates tune the transparency of the weak links while the plunger gate voltage $V_{P G}$ tunes the chemical potential of the island, and the global back-gate voltage $V_{B G}$ tunes the chemical potential of the whole system. The SCPTs are mounted to the cold finger of a dilution refrigerator with a base temperature of $27 \mathrm{mK}$. We report on six devices in total; in the main text we present data on a device with a $500 \mathrm{~nm}$ long island (see Table S1 of the Supplemental Material for an overview of all the devices [40]). Unless otherwise indicated, the presented data were obtained at $27 \mathrm{mK}$ and at zero magnetic field.

\section{RESULTS}

\section{A. Coulomb blockade and switching current histograms}

We first tune the device into Coulomb blockade by increasing the heights of the barriers separating the island from the leads. The clear, regular Coulomb diamonds shown in Fig. 2(a) demonstrate the creation of a single, well-defined island. Moreover, a 1e-periodic conductance modulation appears when $e\left|V_{b}\right|>4 \Delta$ and transport through the island is dominated by quasiparticles which enables us to identify the gate voltage periodicity corresponding to $1 e$ [41]. The current at lower bias voltages is too small to resolve in the Coulomb blockade regime since it involves Cooper pair transport and is therefore higher order in the tunneling. Finally, we extract the superconducting gap $\Delta=180 \mu \mathrm{eV}$ and the geometric charging energy $E_{C}^{0}=1.5 \mathrm{meV}$ from the observed diamonds.

In order to generate a measurable supercurrent, we lower the barriers in order to increase $E_{J}$ which simultaneously suppresses $\delta E_{\mathrm{eo}}$. The switching current is recorded by triggering on the voltage step in the $I-V$ curve as illustrated in Fig. 2(c); this is repeated $N$ times for each $n_{g}$ to gather statistics, typically $N=100$ to 500 . Figure 2(b) shows the resulting switching current histogram which is $2 e$ periodic, indicating that in this regime the charge dispersion has decreased at least an order of magnitude to the point that $\delta E_{\mathrm{eo}}<\Delta$, consistent with the observed charging energy renormalization in a nanowire island with normal leads [16].

To establish that our observed $2 e$ periodicity is robust, we investigate the gate-charge modulation for a wide range of gate settings, as is shown in Fig. 2(d). We characterize each gate setting by the normal state resistance of the device. Figure 2(d) shows that the modulation is observed for $R_{N}$ ranging from 5.8 to $19.6 \mathrm{k} \Omega$. At $R_{N}=5.8 \mathrm{k} \Omega$, the switching current was only modulated by $5 \%$, indicating that the device is in the Josephson dominated regime where $E_{J}>\delta E_{\mathrm{eo}}$. 

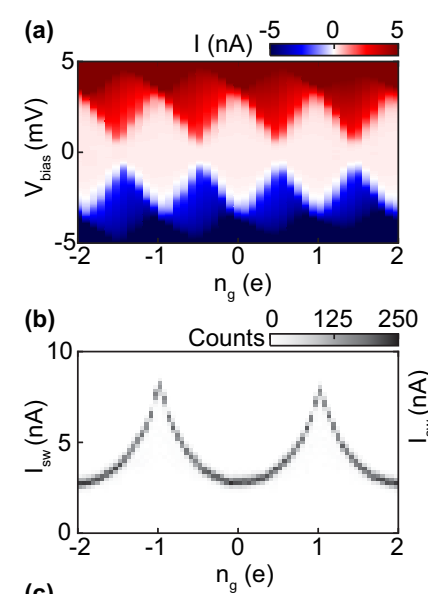

(c)
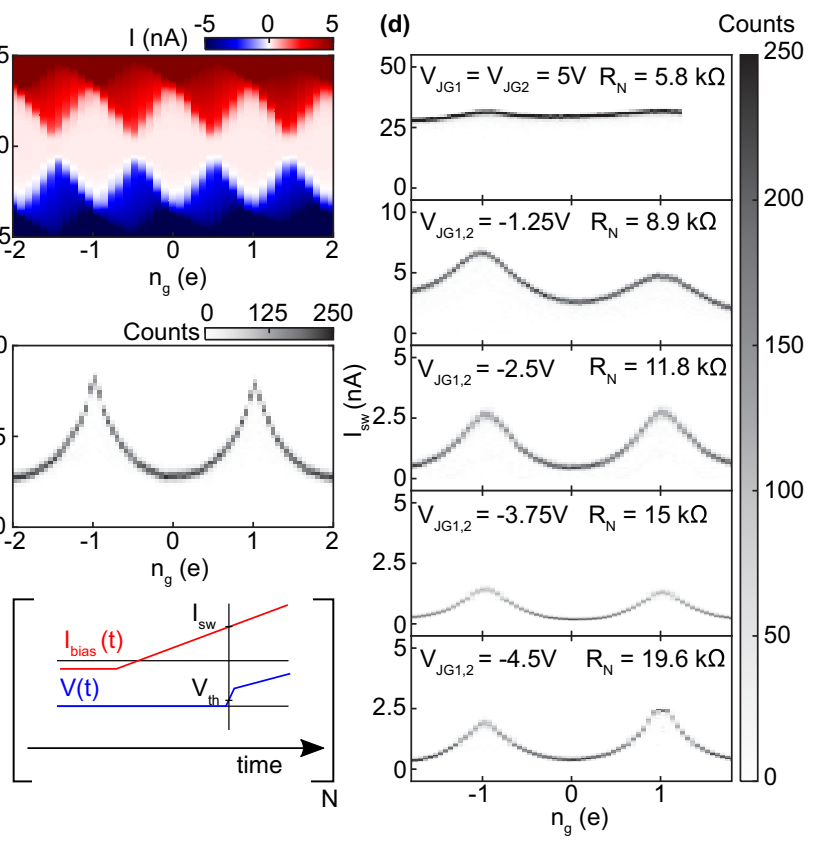

FIG. 2. Gate dependence of the $2 e$-periodic switching current modulation. (a) Charge stability diagram measured in the strongly Coulomb-blockaded regime with $\Delta=180 \mu \mathrm{eV}$ and $E_{C}^{0}=1.5 \mathrm{meV}$. (b) Histogram of the $2 e$-periodic switching current $I_{s w}$ in the weakly Coulomb-blockaded regime, indicating that $\Delta>\delta E_{\mathrm{eo}}$. At this gate setting, $V_{J G 1}=-4.1 \mathrm{~V}$ and $V_{J G 2}=-5.7 \mathrm{~V}, R_{N}=14.8 \mathrm{k} \Omega$. (c) Schematic representation of the current ramp (in red) used to obtain the $I_{s w}$ histograms and the resulting voltage across the SCPT (in blue). The switching current $I_{s w}$ is recorded when the voltage drop on the SCPT reaches a threshold value $V_{\text {th }}$. (d) Switching current histograms for varying normal state resistance. The normal state resistance is calculated as the average over the $n_{g}$ range at high bias. Note the change of vertical scale for the two topmost panels. The peak height asymmetry seen for $R_{N}=8.9 \mathrm{k} \Omega$ and $R_{N}=19.6 \mathrm{k} \Omega$ is due to cross coupling between the junctions and $V_{P G}$.

The other devices behave similarly as can be seen in Fig. S1 of the Supplemental Material [40]. Five out of the six measured SCPTs show a $2 e$-period modulation robust over different gate settings. The remaining SCPT (device 5) exhibits an even-odd pattern, indicating that $\delta E_{\mathrm{eo}}>\Delta$. Nevertheless, the robustness of the $2 e$ signal across gate settings and devices suggests a general lack of low-energy subgap states inside the islands at zero field, consistent with the hard gap observed in bias spectroscopy experiments which locally probe the density of states $[37,42]$.

\section{B. Temperature dependence and modeling}

To gain insight into the relevant poisoning mechanisms of the SCPT, we measure the temperature dependence of the $2 e$-periodic switching current modulation at $R_{N}=14.8 \mathrm{k} \Omega$. As can be seen in Fig. 3(a), we observe that the $2 e$ periodicity persists up to $T \approx 189 \mathrm{mK}$ at which point the oscillations develop local maxima at even $n_{g}$ values and finally become fully $1 e$ periodic for $T^{*} \approx 300 \mathrm{mK}$. This is consistent with an expected level spacing of the $\mathrm{Al}$ shell $\delta$ of a few $\mathrm{mK}$ when using the estimate for vanishing charge dispersion $k_{B} T^{*}=$
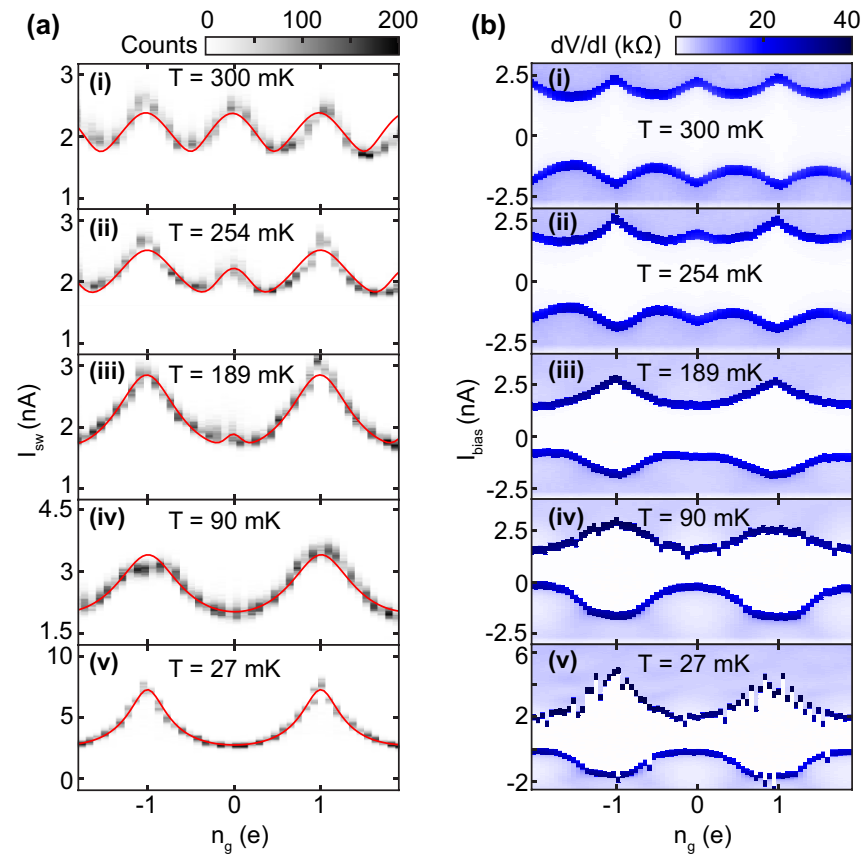

FIG. 3. Temperature dependence. (a) Switching current modulation as a function of temperature. The experimental histograms shown in grayscale are overlaid by the theoretical fit to the average switching current $\left\langle I_{\mathrm{sw}}\right\rangle$ (red curves). Individual fits are for different values of $\Delta, E_{J}$, and $E_{C}$. The average values for resulting the parameters are $\Delta \approx 220 \mu \mathrm{eV}, E_{J} \approx 43 \mu \mathrm{eV}$, and $E_{C} \approx 160 \mu \mathrm{eV}$. (b) $d V / d I$ data for the same temperature range as in (a) obtained from numerical derivation of the $I-V$ curves. The current bias is swept from negative to positive values, hence, the switching (retrapping) current at positive (negative) bias. At elevated temperatures the overdamped $d V / d I$ data shows a similar behavior as the histograms in (a) with local maxima appearing at even $n_{g}$ at $T \approx 189 \mathrm{mK}$ and a fully $1 e$ periodic modulation at $T^{*} \approx 300 \mathrm{mK}$. At low temperatures the junction is in the underdamped regime as indicated by the asymmetric $d V / d I$ and the increased fluctuations due the absence of self-averaging.

$\Delta / \ln (\Delta / \delta)$ [43]. For comparison to the histograms, Fig. 3(b) shows $d V / d I$ data taken over the same temperature range; linecuts of individual $I-V$ traces are shown in Fig. S2 of the Supplemental Material. At elevated temperatures the $d V / d I$ characteristics show a similar behavior as the histograms including the onset of local maxima at even $n_{g}$. This can be explained by a self-averaging that takes place in the overdamped regime due to a succession of multiple switching and retrapping events. Indeed, we note that for $T>189 \mathrm{mK}$, the $d V / d I$ traces show negligible hysteresis, indicating that the SCPT is in the overdamped regime. At low temperatures the junction enters the underdamped regime where a single phase slip can drive the junction normal, which leads to increased fluctuations in the $d V / d I$ data at base temperature.

Our modeling of the $d V / d I$ data, outlined in Supplemental Material Sec. II [40], focuses on the overdamped regime. We identify two limiting cases, depending on the ratio of the parity switching times controlled by $1 / \Gamma_{\text {in }}, 1 / \Gamma_{\text {out }}$ and the response time of the SCPT given by the Josephson time constant $\tau_{J}=\hbar / 2 e I_{c} R_{J}$ [44], with $R_{J}$ the effective shunt 
resistance of the device and $I_{c}$ the critical current. For slow parity switches one expects a double peak structure in the $d V / d I$. In contrast we observe a parity-averaged single peak in the $d V / d I$ which shows that at high temperatures the SCPT is in the fast parity switching regime $\Gamma_{\text {in }}, \Gamma_{\text {out }} \gg 1 / \tau_{J}$. At $T \approx 189 \mathrm{mK}$ where the SCPT transitions into the overdamped regime, $R_{J} \approx 180 \Omega$ and $I_{c} \approx 3 \mathrm{nA}$ leading to $\tau_{J} \approx 1 \mathrm{~ns}$ in our experiment.

Given the fast (un)poisoning at high temperature, we model the observed switching currents as the weighted sum of the switching current of the even and the odd parity states, with the relative probabilities governed by the free energy difference of the two states. Our model includes the charging energy of the island, Josephson coupling of the island to the leads, and the entropic factor associated with bringing a quasiparticle into the island; see Supplemental Material Sec. III for a more detailed discussion [40]. We note that though the fast (un)poisoning is a necessary assumption to fit the data at high temperature $T>189 \mathrm{mK}$, at low temperatures the probability to find the system in the odd state becomes negligible, i.e., $p_{\text {odd }} / p_{\text {even }} \propto \exp \left(-\left(\Delta-\delta E_{\text {eo }}\right) / k_{B} T\right) \rightarrow 0$ for $\Delta>\delta E_{\text {eo. }}$. Thus, for low temperatures the system is essentially only in the even state which yields the $2 e$-periodic histograms of Fig. 3(a) (v).

The fitting gives approximate values of the $\Delta, E_{J}$, and $E_{C}$. These values have error bars of the order of half of their values due to the weak parameter dependence of the fitting function. The fitted value of the superconducting gap $\Delta \approx 220 \mu \mathrm{eV}$ is, within its error bar, consistent with the value obtained from the Coulomb diamonds in Fig. 2(a). Similarly, the fitted $E_{J} \approx$ $43 \mu \mathrm{eV}$ is consistent with the observed switching current. The fitted effective $E_{C} \approx 160 \mu \mathrm{eV}$, however, is smaller than $E_{C}^{0}$ extracted from the Coulomb diamond data in Fig. 2(a). This indicates that, in the regime of open barriers, $E_{C}$ is significantly renormalized by virtual quasiparticle tunneling processes relative to the geometric charging energy [27-31]. The set of consistent fit parameters, together with an excellent fit of the model to the observed switching current dependence on $n_{g}$, supports the validity of the model and the assumption of fast (un)poisoning at high temperatures. Similar fitting results for device 2 strengthen this conclusion, see Supplemental Material Fig. S5 [40].

\section{Parallel magnetic field dependence}

Next, we study the effect of a parallel magnetic field on the switching current modulation. In particular, we tune the gates such that $R_{N}=12.9 \mathrm{k} \Omega$ and $I_{s w}$ shows a $2 e$-periodic modulation at zero field, as is shown in Fig. 4(b). The $2 e$ periodicity implies that $\Delta>\delta E_{\mathrm{eo}}$ and thus that the ground state is always even. As a magnetic field is applied along the nanowire axis, the spinful, odd-charge states are split by the Zeeman energy, thereby reducing the minimal single-particle excitation energy $E_{0}$ of the island. Here, we consider a bound state with energy $E_{0}$. This state is residing in the island since its energy is modulated by $n_{g}$ [14]. The parity dependence of the bound state energy suggests that its origin is superconductivity related. Moreover, the effective $\mathrm{g}$ factor of a bound state residing partially in the InAs nanowire may be larger than that of the states in the $\mathrm{Al}$ shell $[45,46]$. This is why in Fig. 4(a) the bound state energy is detached from the quasiparticle continuum for finite magnetic fields. Interestingly, when the applied field is large enough so that $E_{0}<\delta E_{\mathrm{eo}}$, the parity of the ground state around $n_{g}= \pm 1$ changes to odd. During the retrapping process of the switching current measurement the system tends to be reset to the ground state, indicated by the general lack of bimodal switching current distributions in our data. Hence, the corresponding parity flip shows up as a dip in the switching current modulation around odd $n_{g}$, causing an even-odd pattern. Figures 4(c) and 4(d) show examples of this even-odd structure in the switching current modulation measured at $250 \mathrm{mT}$ and $300 \mathrm{mT}$, respectively.

We investigate the field dependence of this even-odd pattern in more detail by defining the length in gate charge over which the even (odd) state is stable as $S_{\text {even }}\left(S_{\text {odd }}\right.$ ). In Fig. 4(e) these spacings are tracked as a function of the magnetic field using both switching current histograms and $I-V$ measurements, see Fig. S6 and S7 of the Supplemental Material for the representative data [40]. The even (odd) data points are obtained by averaging over 2 (3) successive spacings. Earlier studies performed in metallic superconducting islands found a monotonous drop in $S_{\text {even }}$ [7-9,47]. In contrast, we find an oscillating behavior in the even and odd spacings with the first crossing at $420 \mathrm{mT}$. After the first crossing, the spacings oscillate around $1 e$ with increasing oscillation amplitude. The crossings indicate a closing and reopening of the energy gap for single-particle excitations in the island. Therefore, we conclude that the oscillating pattern is caused by the field-induced zero energy crossings of a single bound state that is detached from the continuum as is illustrated in Fig. 4(a).

Similar to Fig. 3, the histograms and $I$ - $V$ characteristics mostly coincide. For small fields below $200 \mathrm{mT}$, however, the histograms indicate an even ground state, while the slower $I-V$ traces display an even-odd pattern, see Fig. 4(e). This discrepancy occurs because the slower $I-V$ measurements are sensitive to rare trapping events of quasiparticles in the island [33]. The latter occur since even in the absence of subgap states the island acts as a metastable trap with energy $\delta E_{\text {eo }}$ below the gap of the superconducting lead around odd $n_{g}$. In rare cases the metastable state becomes occupied long enough by quasiparticles to cause switching to the resistive state.

In addition, we measure the parity lifetime of the SCPT in a parallel field by performing slow histogram measurements while fixing the gate charge at $n_{g}=1$ so that the extracted lifetime corresponds to poisoning of the even state $[11,38]$. For representative histograms see the lower inset of Fig. 4(f) and Fig. S8 of the Supplemental Material [40]. At $n_{g}=1$, we expect the worst-case scenario for poisoning since the energy difference between the even and odd state is maximal (i.e., favoring the odd state). We observe that this lifetime decreases exponentially with field between 225 and $300 \mathrm{mT}$, see Fig. 4(f). We are limited to this intermediate field range because the lifetime is too large to obtain useful statistics at lower fields and too small to be captured by the bandwidth of the measurement electronics at larger fields. Still, by extrapolating the lifetime to $415 \mathrm{mT}$ where $S_{\text {even }}=S_{\text {odd }}=1 e$, one can estimate the parity lifetime when the bound state is at zero energy to be $\approx 1$ ns. 


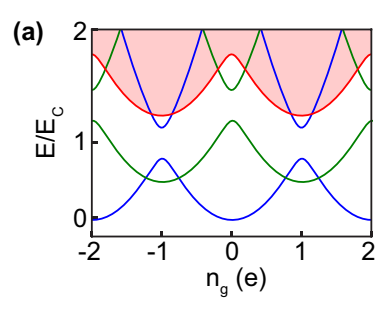

(b)

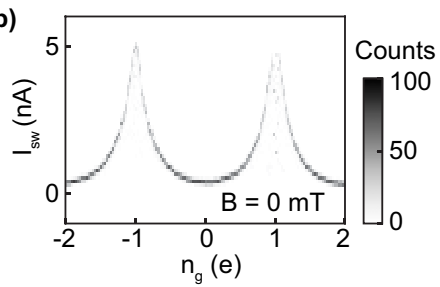

(e)

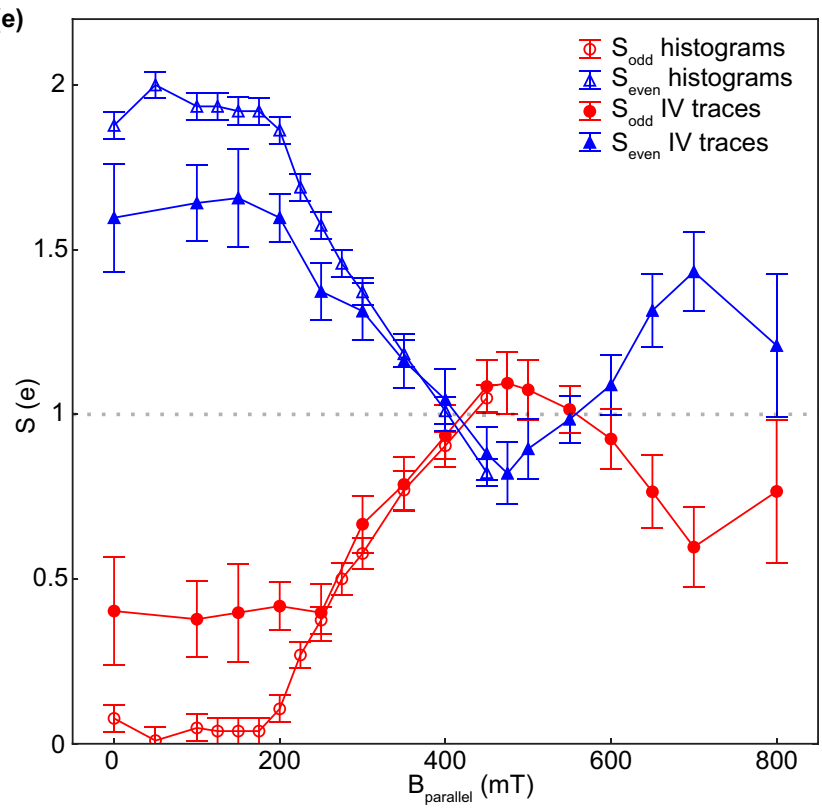

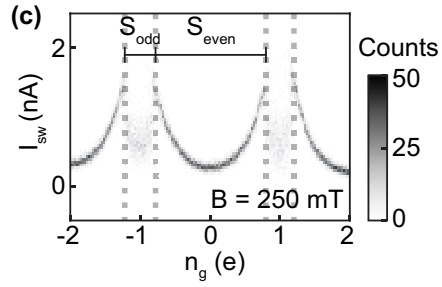

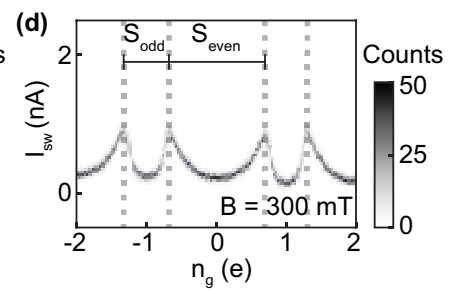

(f)

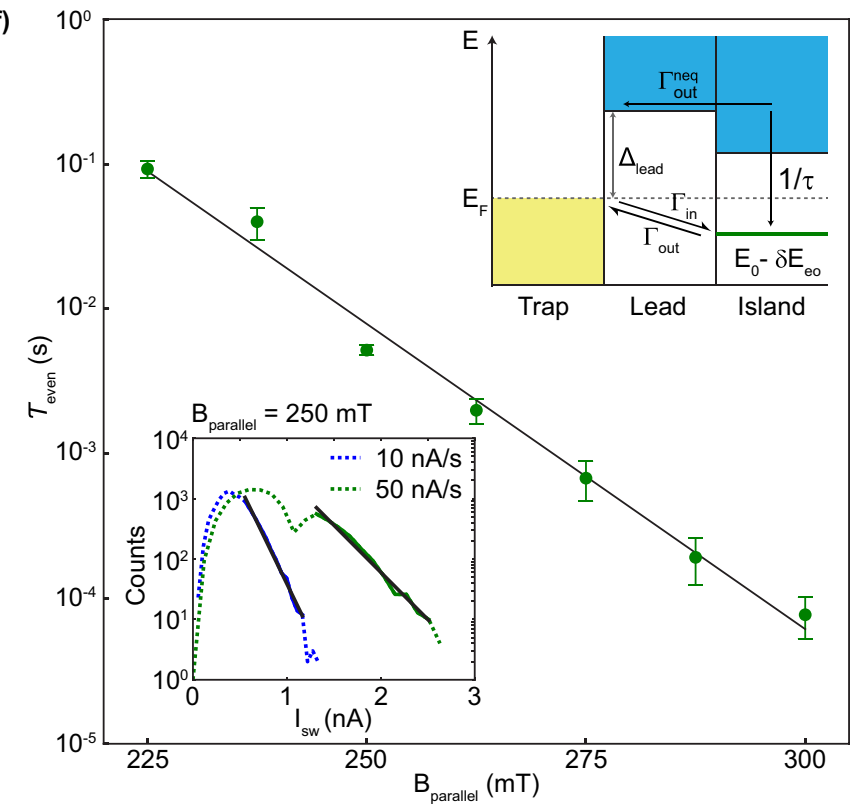

FIG. 4. Parallel magnetic field dependence. (a) The many body energy levels of the SCPT at finite magnetic field. The blue lines indicate the ground and first excited state of the even parity branch. The odd parity ground state is shown in red with the shaded red region emphasizing the quasiparticle continuum on the island. The green curves indicate the presence of a bound state on the island with energy $E_{0}(B)$ leading to an even-odd structure of the ground state when $E_{0}<\delta E_{\mathrm{eo}}$. (b)-(d) Switching current histograms at $0 \mathrm{mT}, 250 \mathrm{mT}$, and $300 \mathrm{mT}$ showing the field evolution of the even-odd structure. (e) The even and odd spacings as a function of the parallel magnetic field obtained from both histograms and $I-V$ traces. The observed crossing at $420 \mathrm{mT}$ and subsequent oscillation is attributed to the presence of a bound state that oscillates about zero energy as a function of magnetic field while the superconducting bulk on the island remains gapped. For the most part switching current histograms and $I-V$ characteristics give the same spacings. At low fields the slow $I-V$ measurements pick up rare poisoning events and thus do not recover full $2 e$ periodicity. (f) The even parity lifetime at $n_{g}=1$ as a function of the magnetic field. The solid line is a guide to the eye indicating an exponential dependence. The lower inset presents a typical dataset used for the extraction of $\tau_{\text {even }}$. The upper inset shows a schematic representation of the energy needed to add a single quasiparticle to different parts of the device.

\section{DISCUSSION}

We begin by noting that the growth of the even-odd spacing oscillation as a function of field seen in Fig. 4(e) is reminiscent of one of the proposed signatures of overlapping Majorana zero modes [48]. However, this increasing oscillation amplitude was only observed in a narrow gate range in our device, as is illustrated in Fig. S9 of the Supplemental Material [40]. This makes it difficult to map the amplitude of the first oscillation to a Majorana overlap, as was done in Ref. [15]. From our results we can only conclude that if this oscillation is indeed due to the presence of overlapping MZMs, the topological portion of the device parameter space is rather small. Nevertheless, mapping the even-odd peak spacing in this manner could be used in future experiments to signal the transition to the topological regime in devices with superconducting leads such as the ones proposed in Refs. [24-26]. This could be an attractive alternative to gap-edge spectroscopy
[49-52] as a signature of the topological regime in these all-superconducting systems.

We also note that the splitting of the $2 e$ signal into an (oscillating) even-odd signal is not always observed. Measurements performed on device 4 , which has a $3 \mu \mathrm{m}$-long island, show a sharp transition of the $2 e$ signal to the $1 e$ signal at a parallel field of $100 \mathrm{mT}$, similar to the behavior observed while increasing the temperature in device 1, see Fig. S10 in the Supplemental Material [40]. This field evolution of the $I_{s w}$ modulation indicates that the SCPT is in the fast (un)poisoning limit with $\Gamma_{\text {in }} / \Gamma_{\text {out }} \approx 1$, possibly caused by a field-induced softening of the superconducting gap in the island and/or leads.

To understand the exponential decrease of the even state lifetime with field seen in Fig. 4(f), we model the system as an island connected to a gapped superconducting lead in contact with a normal metal quasiparticle trap as is shown in the upper inset of Fig. 4(f). In the field range where we 
measure the lifetime, the observed even-odd pattern indicates that the energy difference between the odd and even state at $n_{g}=1$ is always negative, as also depicted in Fig. 4(a) and the inset of Fig. 4(f). Therefore, at $n_{g}=1$ poisoning is only prevented by the quasiparticle filtering effect of the superconducting gap in the leads. Quasiparticles can cross this gap in two ways: by thermal excitation to the gap edge or by tunneling through the gap. Both processes are exponentially suppressed by a factor that scales with the size of the gap in the leads $\Delta_{\text {lead }}$. However, quantitative estimates of the relative strength of the tunneling and thermal activation contributions require a microscopic knowledge of the device. Still, both processes lead to an exponential dependence of the lifetime with field since $\Delta_{\text {lead }}(B)=\Delta_{\text {lead }}(0)-\frac{1}{2} g \mu_{B} B$. In either case, the filtering effect should be enhanced by increasing the length of the superconducting leads as well as by increasing $\Delta_{\text {lead }}$. Since recent studies indicate that the size of the proximitized gap in semiconducting nanowires is gate tunable $[45,46]$, we suggest enhancing this filtering effect by locally gating the leads of the SCPT. Additionally, the length of the leads could be varied in order to investigate the proximity effect of the traps on the gap in the leads $[53,35]$.

Next, for a Majorana-based qubit one is primarily concerned with poisoning events which change the state of the qubit-namely, poisoning of the MZMs [54]. If direct tunneling from the quasiparticle trap is the dominant poisoning mechanism, the subgap state is expected to be directly poisoned since it is the lowest energy state on the island. In this case, the measured $\tau_{\text {even }}$ in Fig. 4(f) directly gives the bound state lifetime since the quasiparticle residence time in the subgap state is likely to be longer than the relevant switching timescale of the junction- $\tau_{J}$ in the case of an overdamped junction and $2 \pi / \omega_{p}$ where $\omega_{p}$ is the plasma frequency in the case of an underdamped junction. In the opposite case of thermally activated poisoning, quasiparticles are first transferred elastically from the superconducting lead to the continuum in the island before relaxing to the subgap state within a time $\tau$ [33]. In this case, quasiparticles can escape from the island before they are detected if $\Gamma_{\text {out }}^{\text {neq }}$ is faster than the SCPT response time. Note, however, that as long as quasiparticles can be detected faster than $\tau$, most of the poisoning events of the subgap states will be detected. The time $\tau_{\text {even }}$ therefore again represents the parity lifetime of the subgap states while the overall parity of the island might fluctuate faster. Our previous estimate of $\tau_{J} \approx 1 \mathrm{~ns}$ sets a lower bound on our poisoning detection bandwidth since the junction would switch even faster to the resistive state in the underdamped case which we observe at low temperature. Given that typical resonators in time-domain RF measurements have bandwidths of no more than a few 10 s of $\mathrm{MHz}$ $[12,34,35]$, switching current measurements are a promising alternative before Majorana poisoning times can be measured more directly via the coherence of MZM-based qubits.

Finally, with the design of future MZM-based qubits in mind it is worth comparing our results with those obtained with NbTiN islands [38]. Our observed gate-charge modulation of the switching current shows a robust $2 e$-periodic signal for a wide range of gate settings which indicates that there are no low-energy subgap states inside the SCPTs at zero magnetic field. This is in stark contrast to the case of $\mathrm{NbTiN}$ islands, where subgap states result in a $1 e$-periodic, bimodal switching current distribution. In that case, despite the large superconducting gap, the island parity is effectively randomized after each measurement when the island retraps after being flooded with quasiparticles.

\section{CONCLUSION}

We have investigated quasiparticle poisoning in hybrid InAs-Al SCPTs by measuring the gate charge modulation of the switching current as a function of temperature and magnetic field. In contrast to previous studies of NbTiN SCPTs, we observe a consistent $2 e$-periodic supercurrent at zero field despite having a similar gap in the island and leads. This highlights the fact that at zero field there are no subgap states in the island and places $\mathrm{Al}$ as the superconductor of choice for MZM qubit experiments despite its smaller gap and critical field relative to NbTiN. In addition, we have observed an oscillating pattern in the gate periodicity of the supercurrent due to the field-induced zero energy crossing of a bound state. This opens the door to using the switching current to identify MZMs in qubit devices with superconducting leads. This is a crucial proof-of-principle demonstration as the superconducting leads are not compatible with the zero-bias peak measurements typically taken as evidence of MZMs. We have performed lifetime measurements on this subgap state and observed an exponential decay of the lifetime in magnetic field due to a collapsing filtering effect of the leads. This exponential decay highlights the importance of proper engineering of the superconducting gap via local gating and intentional quasiparticle traps to minimize the presence of quasiparticles in the leads in future topological qubits.

\section{ACKNOWLEDGMENTS}

The authors thank D. J. van Woerkom for useful discussions and R. N. Schouten and O. Benningshof for technical assistance. This work has been supported by the Netherlands Organization for Scientific Research (NWO), Microsoft Corporation Station Q, the Danish National Research Foundation, and a Synergy Grant of the European Research Council.
[1] K. A. Matveev, M. Gisselfält, L. I. Glazman, M. Jonson, and R. I. Shekhter, Parity-Induced Suppression of the Coulomb Blockade of Josephson Tunneling, Phys. Rev. Lett. 70, 2940 (1993).

[2] D. V. Averin and Y. V. Nazarov, Single-Electron Charging of a Superconducting Island, Phys. Rev. Lett. 69, 1993 (1992).
[3] H. Grabert and M. H. Devoret, Single Charge Tunneling: Coulomb Blockade Phenomena in Nanostructures (Springer Science + Business Media, New York, 2013), Vol. 294.

[4] D. V. Averin and K. K. Likharev, Coulomb blockade of singleelectron tunneling, and coherent oscillations in small tunnel junctions, J. Low Temp. Phys. 62, 345 (1986). 
[5] L. J. Geerligs, V. F. Anderegg, J. Romijn, and J. E. Mooij, Single Cooper-Pair Tunneling in Small-Capacitance Junctions, Phys. Rev. Lett. 65, 377 (1990).

[6] M. T. Tuominen, J. M. Hergenrother, T. S. Tighe, and M. Tinkham, Experimental Evidence for Parity-Based 2e Periodicity in a Superconducting Single-Electron Tunneling Transistor, Phys. Rev. Lett. 69, 1997 (1992).

[7] P. Joyez, P. Lafarge, A. Filipe, D. Esteve, and M. H. Devoret, Observation of Parity-Induced Suppression of Josephson Tunneling in the Superconducting Single Electron Transistor, Phys. Rev. Lett. 72, 2458 (1994).

[8] P. Lafarge, P. Joyez, D. Esteve, C. Urbina, and M. H. Devoret, Measurement of the Even-Odd Free-Energy Difference of an Isolated Superconductor, Phys. Rev. Lett. 70, 994 (1993).

[9] P. Lafarge, P. Joyez, D. Esteve, C. Urbina, and M. H. Devoret, Two-electron quantization of the charge on a superconductor, Nature (London) 365, 422 (1993).

[10] T. M. Eiles and J. M. Martinis, Combined josephson and charging behavior of the supercurrent in the superconducting single-electron transistor, Phys. Rev. B 50, 627 (1994).

[11] J. Männik and J. E. Lukens, Effect of Measurement on the Periodicity of the Coulomb Staircase of a Superconducting Box, Phys. Rev. Lett. 92, 057004 (2004).

[12] O. Naaman and J. Aumentado, Time-domain measurements of quasiparticle tunneling rates in a single-Cooper-pair transistor, Phys. Rev. B 73, 172504 (2006).

[13] A. J. Ferguson, N. A. Court, F. E. Hudson, and R. G. Clark, Microsecond Resolution of Quasiparticle Tunneling in the SingleCooper-Pair Transistor, Phys. Rev. Lett. 97, 106603 (2006).

[14] A. P. Higginbotham, S. M. Albrecht, G. Kiršanskas, W. Chang, F. Kuemmeth, P. Krogstrup, T. S. Jespersen, J. Nygård, K. Flensberg, and C. M. Marcus, Parity lifetime of bound states in a proximitized semiconductor nanowire, Nat. Phys. 11, 1017 (2015)

[15] S. M. Albrecht, A. P. Higginbotham, M. Madsen, F. Kuemmeth, T. S. Jespersen, J. Nygård, P. Krogstrup, and C. M. Marcus, Exponential protection of zero modes in Majorana islands, Nature (London) 531, 206 (2016).

[16] D. Sherman, J. S. Yodh, S. M. Albrecht, J. Nygård, P. Krogstrup, and C. M. Marcus, Normal, superconducting and topological regimes of hybrid double quantum dots, Nat. Nanotechnol. 12, 212 (2017).

[17] S. M. Albrecht, E. B. Hansen, A. P. Higginbotham, F. Kuemmeth, T. S. Jespersen, J. Nygård, P. Krogstrup, J. Danon, K. Flensberg, and C. M. Marcus, Transport Signatures of Quasiparticle Poisoning in a Majorana Island, Phys. Rev. Lett. 118, 137701 (2017).

[18] J. Shen, S. Heedt, F. Borsoi, B. Van Heck, S. Gazibegovic, R. L. M. Veld, D. Car, J. A. Logan, M. Pendharkar, G. Wang et al., Parity transitions in the superconducting ground state of hybrid InSb-Al Coulomb islands, arXiv:1804.08405.

[19] E. C. T. O'Farrell, A. C. C. Drachmann, M. Hell, A. Fornieri, A. M. Whiticar, E. B. Hansen, S. Gronin, G. C. Gardener, C. Thomas, M. J. Manfra, K. Flensberg, C. M. Marcus, and F. Nichele, Hybridization of sub-gap states in onedimensional superconductor/semiconductor Coulomb islands, arXiv:1804.09676.

[20] L. Fu, Electron Teleportation Via Majorana Bound States in a Mesoscopic Superconductor, Phys. Rev. Lett. 104, 056402 (2010).
[21] B. van Heck, R. M. Lutchyn, and L. I. Glazman, Conductance of a proximitized nanowire in the Coulomb blockade regime, Phys. Rev. B 93, 235431 (2016).

[22] B. Van Heck, A. R. Akhmerov, F. Hassler, M. Burrello, and C. W. J. Beenakker, Coulomb-assisted braiding of Majorana fermions in a Josephson junction array, New J. Phys. 14, 035019 (2012).

[23] T. Hyart, B. van Heck, I. C. Fulga, M. Burrello, A. R. Akhmerov, and C. W. J. Beenakker, Flux-controlled quantum computation with Majorana fermions, Phys. Rev. B 88, 035121 (2013).

[24] D. Aasen, M. Hell, R. V. Mishmash, A. P. Higginbotham, J. Danon, M. Leijnse, T. S. Jespersen, J. A. Folk, C. M. Marcus, K. Flensberg et al., Milestones Toward Majorana-Based Quantum Computing, Phys. Rev. X 6, 031016 (2016).

[25] M. Hell, J. Danon, K. Flensberg, and M. Leijnse, Time scales for Majorana manipulation using Coulomb blockade in gatecontrolled superconducting nanowires, Phys. Rev. B 94, 035424 (2016).

[26] D. Litinski, M. S. Kesselring, J. Eisert, and F. von Oppen, Combining Topological Hardware and Topological Software: Color-Code Quantum Computing with Topological Superconductor Networks, Phys. Rev. X 7, 031048 (2017).

[27] V. Ambegaokar, U. Eckern, and G. Schön, Quantum Dynamics of Tunneling Between Superconductors, Phys. Rev. Lett. 48, 1745 (1982).

[28] A. I. Larkin and Y. N. Ovchinnikov, Decay of the supercurrent in tunnel junctions, Phys. Rev. B 28, 6281 (1983).

[29] U. Eckern, G. Schön, and V. Ambegaokar, Quantum dynamics of a superconducting tunnel junction, Phys. Rev. B 30, 6419 (1984).

[30] R. M. Lutchyn, Effect of quantum fluctuations on even-odd energy difference in a Cooper-pair box, Phys. Rev. B 75, 212501 (2007).

[31] R. M. Lutchyn, K. Flensberg, and L. I. Glazman, Quantum charge fluctuations of a proximitized nanowire, Phys. Rev. B 94, 125407 (2016).

[32] J. Aumentado, M. W. Keller, J. M. Martinis, and M. H. Devoret, Nonequilibrium Quasiparticles and $2 e$ Periodicity in SingleCooper-Pair Transistors, Phys. Rev. Lett. 92, 066802 (2004).

[33] R. M. Lutchyn and L. I. Glazman, Kinetics of quasiparticle trapping in a Cooper-pair box, Phys. Rev. B 75, 184520 (2007).

[34] M. D. Shaw, R. M. Lutchyn, P. Delsing, and P. M. Echternach, Kinetics of nonequilibrium quasiparticle tunneling in superconducting charge qubits, Phys. Rev. B 78, 024503 (2008).

[35] N. A. Court, A. J. Ferguson, R. Lutchyn, and R. G. Clark, Quantitative study of quasiparticle traps using the single-Cooper-pair transistor, Phys. Rev. B 77, 100501 (2008).

[36] P. Krogstrup, N. L. B. Ziino, W. Chang, S. M. Albrecht, M. H. Madsen, E. Johnson, J. Nygård, C. M. Marcus, and T. S. Jespersen, Epitaxy of semiconductor-superconductor nanowires, Nat. Mater. 14, 400 (2015).

[37] W. Chang, S. M. Albrecht, T. S. Jespersen, F. Kuemmeth, P. Krogstrup, J. Nygård, and C. M. Marcus, Hard gap in epitaxial semiconductor-superconductor nanowires, Nat. Nanotechnol. 10, 232 (2015).

[38] D. J. Van Woerkom, A. Geresdi, and L. P. Kouwenhoven, One minute parity lifetime of a NbTiN Cooper-pair transistor, Nat. Phys. 11, 547 (2015). 
[39] K. M. Lang, S. Nam, J. Aumentado, C. Urbina, and J. M. Martinis, Banishing quasiparticles from Josephson-junction qubits: Why and how to do it, IEEE Trans. Appl. Supercond. 13, 989 (2003).

[40] See Supplemental Material at http://link.aps.org/supplemental/ 10.1103/PhysRevB.98.174502 for additional data and a detailed discussion of the used models.

[41] P. Hadley, E. Delvigne, E. H. Visscher, S. Lähteenmäki, and J. E. Mooij, $3 e$ tunneling processes in a superconducting singleelectron tunneling transistor, Phys. Rev. B 58, 15317 (1998).

[42] M. T. Deng, S. Vaitiekenas, E. B. Hansen, J. Danon, M. Leijnse, K. Flensberg, J. Nygård, P. Krogstrup, and C. M. Marcus, Majorana bound state in a coupled quantum-dot hybrid-nanowire system, Science 354, 1557 (2016).

[43] K. A. Matveev, L. I. Glazman, and R. I. Shekhter, Effects of charge parity in tunneling through a superconducting grain, Mod. Phys. Lett. B 08, 1007 (1994).

[44] R. L. Kautz and J. M. Martinis, Noise-affected I-V curves in small hysteretic Josephson junctions, Phys. Rev. B 42, 9903 (1990).

[45] S. Vaitiekėnas, M.-T. Deng, J. Nygård, P. Krogstrup, and C. M. Marcus, Effective $g$ Factor of Subgap States in Hybrid Nanowires, Phys. Rev. Lett. 121, 037703 (2018).

[46] M. W. A. de Moor, J. D. S. Bommer, D. Xu, G. W. Winkler, A. E. Antipov, A. Bargerbos, G. Wang, N. van Loo, R. L. M. Op het Veld, S. Gazibegovic, D. Car, J. A. Logan, M. Pendharkar, J. S. Lee, E. P. A. M. Bakkers, C. J. Palmstrøm, R. M. Lutchyn, L. P. Kouwenhoven, and H. Zhang, Electric field tunable superconductor-semiconductor coupling in Majorana nanowires, arXiv:1806.00988.

[47] M. T. Tuominen, J. M. Hergenrother, T. S. Tighe, and M. Tinkham, Even-odd electron number effects in a small superconducting island: Magnetic-field dependence, Phys. Rev. B 47, 11599 (1993).

[48] S. Das Sarma, J. D. Sau, and T. D. Stanescu, Splitting of the zero-bias conductance peak as smoking gun evidence for the existence of the Majorana mode in a superconductorsemiconductor nanowire, Phys. Rev. B 86, 220506 (2012).

[49] F. Setiawan, W. S. Cole, J. D. Sau, and S. Das Sarma, Conductance spectroscopy of nontopological-topological superconductor junctions, Phys. Rev. B 95, 020501 (2017).

[50] P. San-Jose, J. Cayao, E. Prada, and R. Aguado, Multiple Andreev reflection and critical current in topological superconducting nanowire junctions, New J. Phys. 15, 075019 (2013).

[51] S. Nadj-Perge, I. K. Drozdov, J. Li, H. Chen, S. Jeon, J. Seo, A. H. MacDonald, B. A. Bernevig, and A. Yazdani, Observation of Majorana fermions in ferromagnetic atomic chains on a superconductor, Science 346, 602 (2014).

[52] M. Ruby, F. Pientka, Y. Peng, F. von Oppen, B. W. Heinrich, and K. J. Franke, End States and Subgap Structure in ProximityCoupled Chains of Magnetic Adatoms, Phys. Rev. Lett. 115, 197204 (2015).

[53] A. Hosseinkhani and G. Catelani, Proximity effect in normalmetal quasiparticle traps, Phys. Rev. B 97, 054513 (2018).

[54] D. Rainis and D. Loss, Majorana qubit decoherence by quasiparticle poisoning, Phys. Rev. B 85, 174533 (2012). 